\title{
Skill Acquisition and Development Issues with Predictable Badminton Feeding Routines
}

\author{
S.M. Smith ${ }^{1, *}$, E. Tasker ${ }^{1}$, E. Paine ${ }^{1}$, T.M. Hughes ${ }^{1}$, C. Heiden ${ }^{1}$, O. Baczala ${ }^{2}$ \\ ${ }^{1}$ Department of Sport, Exercise and Health, University of Winchester, Winchester, UK \\ 2 Westgate Badminton Performance Centre, Winchester, UK \\ *Corresponding author Email: steve.smith@winchester.ac.uk \\ DOI: https://doi.org/10.34256/ijpefs2213 \\ Received: 16-12-2021, Revised: 22-02-2022; Accepted: 23-02-2022; Published: 25-02-2022
}

Abstract: Badminton practice designs contain feeding routines that allow players to practice technical skills. Optimal skill acquisition and development is gained through practice simulating competitive match play rather than approaches centred around mass repetition with low levels of uncertainty. This study aimed to evaluate the biomechanical differences in twelve youth elite UK badminton players in activities with varying levels of predictability. The three experimental trials were handfeed, multifeed and match play trials. Motion capture cameras were used to record participant biomechanical data. Data analysis revealed several significant biomechanical differences between trials. Results showed that an increase in trial unpredictability led to increased depth of the forward lunge shot and a lower racket shuttlecock strike position with greater forward trunk bend. This study showed that visual search behaviour in badminton is a key component of the forward lunge shot. Predictable practice feeding routines may cause poor skill acquisition and development through maladaptive learning behaviours. Coaches are recommended to implement unpredictable feeding practice routines to simulate the visual search behaviour of competitive badminton match play to enhance skill acquisition and development in youth elite badminton players.

Keywords: Visual Search Behaviour, Representative Learning Design, Ecological Dynamics, Practice

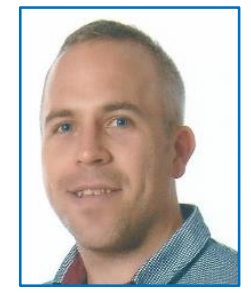

Dr Steve Smith is a Senior Lecturer in Sport Coaching and Psychology and is the Sport Performance Research Lead in the Department of Sport, Exercise and Health at the University of Winchester in the UK. Dr Smith has an extensive knowledge of competing and coaching elite sport.
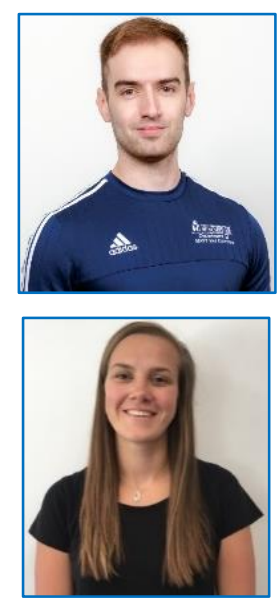

Edward Tasker is the Senior Laboratory Technician at University of Winchester in the UK. Mr Tasker holds an MSc in Applied Sport and Exercise Science.

Eloise Paine is a Senior Sports Science Laboratory Technician at the University of Winchester in the UK. Ms Paine is a PhD candidate exploring pulse wave velocity responses in stroke patients.

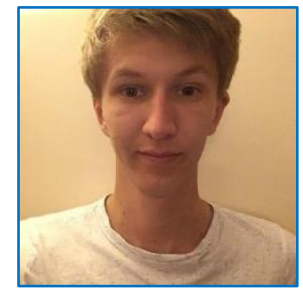

Thomas Hughes is a postgraduate student at the University of Winchester in the UK.

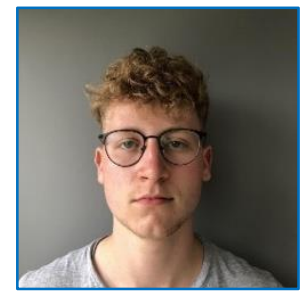

Connor Heiden is an undergraduate student at the University of Winchester in the UK studying BSc Sport and Exercise Psychology.

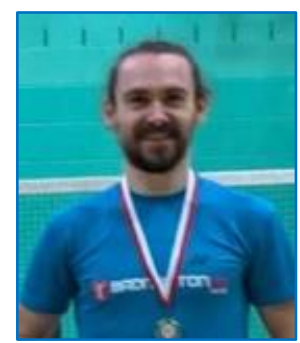

Ollie Baczala is the Head Coach at the Westgate Badminton Performance Centre in the UK. He has competed internationally in badminton and has achieved a world ranking within the top 150. 


\section{Introduction}

Badminton is an extremely demanding sport that requires high-intensity intermittent actions and a high level of anticipatory actions within an uncertain environment [1]. To enhance the skill acquisition of badminton players, the aim of practice is to provide activities that allow individuals the opportunity to acquire innovative and adaptable performance behaviours by simulating competitive play $[2,3]$. Skill acquisition in sport is traditionally centred around mass repetition with low levels of uncertainty, prioritising movement consistency over adaptability [4], despite coach-led sport practice environments being highly individual [5]. There are common badminton practice approaches that include predictable hand thrown and racket shuttlecock feeding routines that are endorsed by the Badminton World Federation (BWF) in their Coach Education Level 2 Award [6]. To enhance badminton practice, recent research has been conducted to develop and optimise automated shuttlecock feeding machines to facilitate badminton activities in the practice environment $[7,8]$. These machines are designed to replicate human shuttlecock feeding and allow an individual to practice technical skills and movements independently. However, as with predictable human feeding, the use of feeding machines in other 'fast ball sports', such as cricket, have shown to cause maladaptive learning behaviours [9].

Ecological psychology [10] and ecological dynamics in sport [11] has led to considerable recent research attention towards representative learning design (RLD) in the sport practice domain. RLD provides a framework proposing training tasks should simulate the necessary sources of contextual information to allow athletes to develop adaptive sportspecific movement solutions, which are more likely to transfer to the competitive environment $[12,13]$. RLD makes the distinction between skill as a technique and skill as an ecological behaviour that is affected by the constraints of the performance environment [14]. For example, badminton practice routines with shuttlecock feeding from a stationary position (low spatial temporal differences) compared to the competitive situation where the shuttlecock is fed from differing locations (high spatial temporal differences). Therefore, a practice environment predicated on RLD will promote action fidelity between practice and competition through task constraints that are representative of the competitive performance context [15] and begs the questions whether there is a place for predictable coach feeding or shuttlecock feeding machines within the practice environment.

Traditional linear learning approaches in sport that contain high levels of predictability through reduction and mass repetition of skill, have been found to be particularly detrimental to the perception-action coupling crucial to skill acquisition and development [11]. A significant reason why predictable learning approaches hamper skill acquisition and development in badminton can be seen with the movements and decisions that players make during badminton matches. Alder and colleagues [16] found badminton movements and decisions were initiated by perceptual cues, suggesting that international badminton players utilise a visual search behaviour (VSB) strategy when making shot decisions (e.g., responses to opponent body position and shuttlecock location). The inclusion of other bodies within the sporting environment has shown skill execution differences in basketball with the addition of a defender [17]. Therefore, the VSB undertaken by badminton players is a vital component of all badminton movements. Practice environments that do not contain the full VSB linked to the badminton movement and decision, may compromise skill acquisition and development.

Badminton research has yet to specifically explore the VSB that players may use during matches, but Wolfe and Horowitz [18] identify several attention guiding attributes to VSB that include motion, distances, orientation, and spatial differences (e.g., location and speed of the shuttlecock and opponent's body position), which vary in unpredictable circumstances in competitive badminton. Previous research [4] found predictable tennis practice tasks failed to replicate the ball (e.g., speed, spin, and trajectory) and movement (e.g., player court position) dynamics of match play, with practice tasks promoting cooperative behaviours rather than combative. Due to the importance of VSB in badminton, the use of feeding routines where the shuttlecock is fed from, and hit to, a predictable location, is not ecologically representative of badminton match play and may limit skill acquisition and development [19].

Currently, to our knowledge, there has been no RLD research conducted to evaluate the technical movement differences between practice activities and match play in badminton. The purpose of this study was to evaluate the biomechanical differences in youth elite badminton players who performed a forward lunge shot (FLS) in activities with varying levels of predictability and VSB. The FLS is a critical movement 
in badminton and was chosen due to its importance and high frequency in singles matches and its technical similarity [20-23]. It was hypothesised that at the moment of racket contact with the shuttlecock, the participants' FLS would be significantly different biomechanically between badminton activities that were predictable compared to unpredictable match play.

\section{Methods}

\subsection{Participants}

Twelve elite youth UK badminton players ( males $=10$; females $=2$; mean age $=14.75 \pm 1.91$ years; mean experience of elite badminton $=5.25 \pm$ 2.22 years; mean weekly training $=10.08 \pm 3.03$ hours) participated in the study. Eleven players were right-handed, and all players were nationally ranked within the top 100 players (ranking range $=1-93$ ). Prior to data collection, all participants were free from injury, completed health screening, and both participant and parent gave written consent. Ethical approval was gained from the authors' university ethics review board.

\subsection{Protocol}

Participants randomly undertook three experimental trials. For each trial, participants were required to play 10 forehand $(\mathrm{FH})$ and 10 backhand (BH) FLS. Eight Oqus motion capture cameras (Qualisys, Gothenburg, Sweden, $460 \mathrm{~Hz} /$ frame) were mounted onto tripods around a badminton court at the venue where all participants undertook regular badminton practice activity (see figure 1 ). Fourteen spherical reflective markers (diameter $=19 \mathrm{~mm}$ ) were placed on each participant at anatomic landmarks that would create a full-body biomechanical model. Reflective markers were placed on acromion processes, lateral epicondyles of the humerus, styloid processes of ulna, anterior-superior iliac spines, lateral condyles of the tibia, lateral malleoli, and second metatarsal heads (see figure 3a) using double-sided sticky tape and kinesiology tape to reinforce the marker position against high speed and impact activity [24].

Reflective tape (width $=10 \mathrm{~mm}$ ) was added to each shuttlecock to capture the moment of the shuttlecock striking the racket head. Four dozen (48) Yonex AS30 feather shuttlecocks were used across all trials for all participants. When shuttlecock flight was compromised through damaged or ruffled feathers, the shuttlecock was replaced. Prior to all trials, the shuttlecocks were flight tested in accordance with BWF tournament regulations.

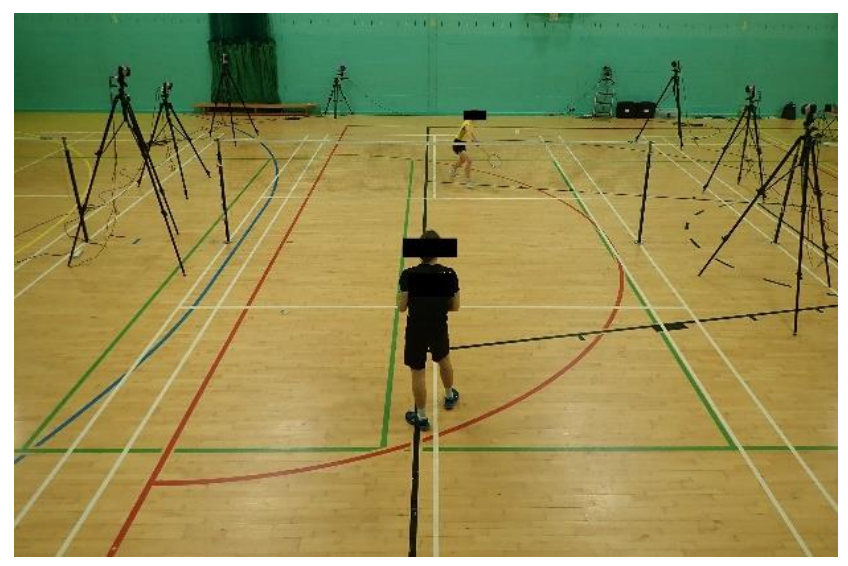

Figure 1 Motion capture camera set up around badminton court

\subsection{Experimental Trials}

All trials were based on singles play in badminton. All participants were familiar with handfeed and multifeed practice routines. High ecological validity was gained by collecting data within participants' usual practice environment. Participants were instructed to use their normal technique during all trials. To enhance reliability in feeding trials, the same highly skilled coach and former international player with seven years coaching experience conducted all feeding. To standardise all movements, participants were instructed to play a straight (not cross court) net shot (forecourt to forecourt) in coach fed trials when the shuttlecock was fed to the forecourt. This was not a requirement for the match play trial where all rallies were recorded until 10 forecourt to forecourt shots were performed for both $\mathrm{FH}$ and $\mathrm{BH}$. Participants were given at least a 2-minute recovery between each trial.

\subsubsection{Handfeed Trial}

The handfeed trial represented a highly predictable practice routine where the participant knew both the starting location and racket strike location of the shuttlecock with low levels of VSB required. The handfeed trial consisted of 10 shuttlecocks thrown by the coach to the participant's FH and BH forecourt area to produce a FLS. Ten shuttlecocks were thrown to one side first and then the other, with a 2-minute recovery period separating each side. $\mathrm{FH}$ and $\mathrm{BH}$ order were randomised for each participant. 


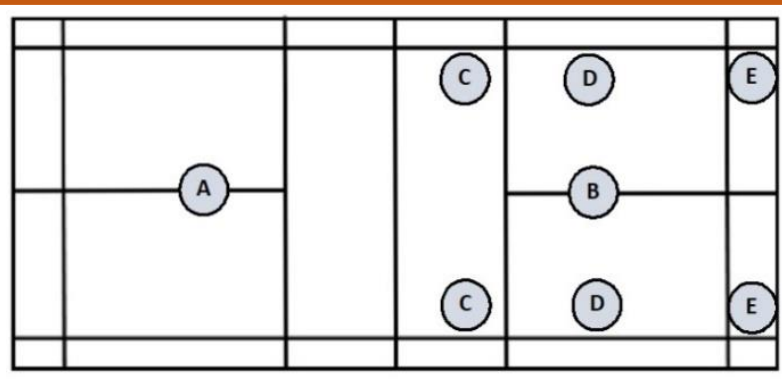

Figure 2 Participant and coach court positions with target feeding areas

The coach stood in position A (central base position on opposite side of court) (see figure 2) and threw shuttlecocks underarm (see figure 3b) over the net to position $C$ (see figure 2). The participant was asked to start their FLS movement from their normal central base at position B (see figure 2), and using a normal FLS technique, return the shuttlecock. Participants would then recover back to their central base position and the coach would feed the next shuttlecock until all 10 were completed.

(a)

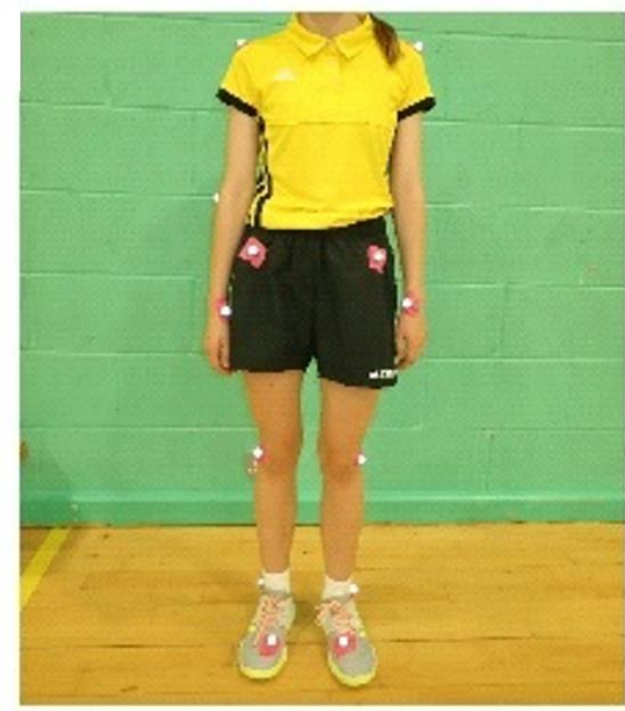

(c)

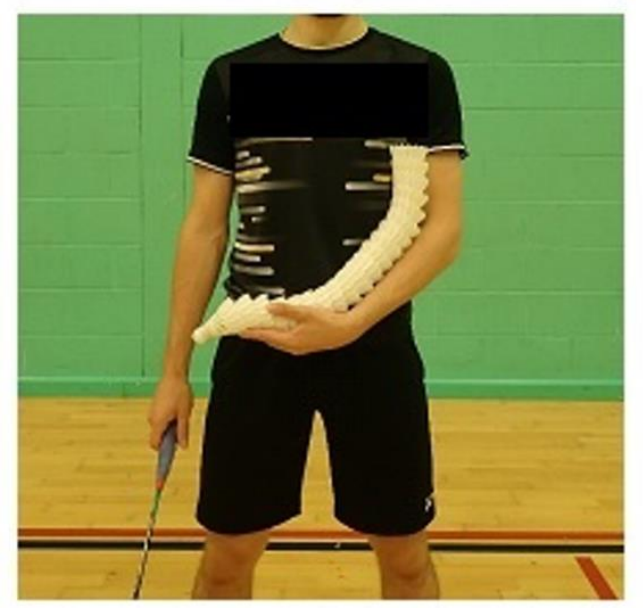

\subsubsection{Multifeed Trial}

The multifeed trial represented a semipredictable practice routine where the participant knew the starting location of the shuttlecock, which required a low level of VSB, but not the location where shuttlecock racket strike would occur. The multifeed trial involved the coach feeding two sets of 20 shuttlecocks from position A (see figure 2) randomly to six areas of the court, which were the forecourt (position C), midcourt (position D), and rearcourt (position E). The coach used a commonplace cradle technique to hold the shuttlecocks (see figure 3c) that allowed for quick release and a FH racket strike from a position anterior, lateral, and inferior to the right hip (see figure 3d). The timing of each shuttlecock feed allowed the participant's FLS to be initiated from the central base position. The coach was instructed to randomly feed 10 shuttlecocks to the forecourt per set of 20 so that by the end of the multifeed trial the participant had made $10 \mathrm{FLS}$ to both the $\mathrm{FH}$ and $\mathrm{BH}$ sides.

(b)

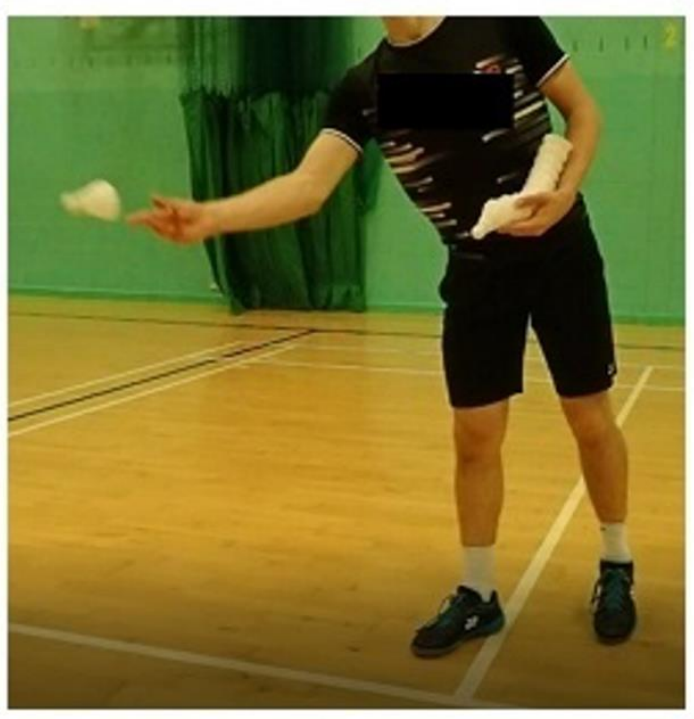

(d)

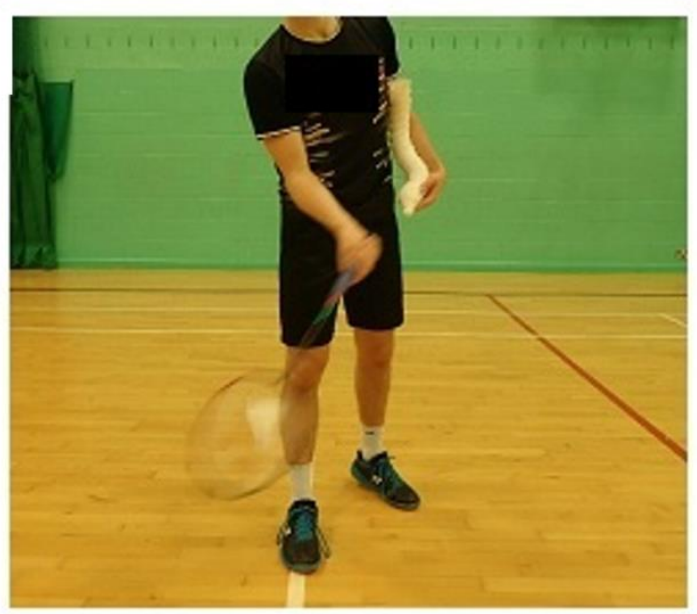

Figure 3 (a) reflective marker placements, (b) coach handfeed throw, (c) coach shuttlecock feeding cradle technique, (d) coach multifeed shuttlecock strike location. 
The coach was supported during the multifeed trial by the lead researcher who kept a tally of shots at the forecourt. To guard against feeder error, the coach held 22 shuttlecocks.

\subsubsection{Match Play Trial}

The feeding and racket strike location of the shuttlecock was unpredictable in the match play trial, which represented the VSB of competitive badminton. Participants played against an opponent of similar ability in accordance with the rules of badminton stated by the BWF. The match continued until the participant recorded $10 \mathrm{FH}$ and $10 \mathrm{BH}$ FLS that initiated from a central base position.

\subsection{Data Analysis}

Biomechanical analysis was performed on the first six (10 shots recorded to guard against potential error in marker pick up by the cameras) readable $\mathrm{FH}$ and BH FLS for all trials using Qualisys Track Manager ver. 2021 (Qualisys, Gothenburg, Sweden). Measurements were taken from the frame when the shuttlecock changed its direction to indicate the moment of racket strike. In total, 432 FLS were analysed. Dominant side (e.g., right arm and leg of right-handed player) readings were taken on key FLS kinematics [21, 25], which included wrist height, shoulder height, trunk angle, hip joint angle, knee joint angle, wrist velocity, knee velocity, ankle velocity, and knee joint angular velocity. Means were calculated for each trial and analysed using IBM SPSS Statistics (Version 27). A one-way repeated measures ANOVA examined intra-individual differences between each trial. Pairwise comparisons were calculated with Bonferroni-adjustments to control for type 1 error inflation typically associated with multiple comparisons. Mauchly's test of sphericity was used to identify violations in the assumption of sphericity, with Greenhouse-Geisser corrections made to the F-statistic when sphericity violations were identified. Significance level was set to 0.05 .

\section{Results}

Mean trial scores and one-way repeated measures ANOVA results with comparisons between trials can be found in table 1 . The intra-individual analysis revealed significant differences between trials in both FH and BH FLS in wrist height, shoulder height, trunk angle, hip joint angle, knee joint angle, and wrist velocity. Results were fairly consistent between $\mathrm{FH}$ and BH FLS trials.

Results showed a linear relationship existed as trials became less predictable. No significant differences were found for knee velocity, ankle velocity, and knee joint angular velocity. $\mathrm{FH}$ wrist height, $\mathrm{BH}$ wrist height, $\mathrm{FH}$ shoulder height and $\mathrm{BH}$ shoulder height all decreased with increasing unpredictability. Trunk angle (forward lean) significantly increased on both $\mathrm{FH}$ and $\mathrm{BH}$ sides as the trials increased in unpredictability. $\mathrm{FH}$ hip joint angle significantly decreased with greater unpredictability, but $\mathrm{BH}$ hip joint angle only decreased significantly between the handfeed trial and the multifeed and match play trials respectively. Knee joint angle means decreased with increasing unpredictability but only showed significant differences between the match play trial and the handfeed and multifeed trials for $\mathrm{FH}$, and for handfeed trial and multifeed and match play trials for $\mathrm{BH}$ respectively. Wrist velocity difference for both $\mathrm{FH}$ and $\mathrm{BH}$ were significantly different only between handfeed and match play trials.

\section{Discussion}

This is the first known study to assess ecological dynamics in a badminton practice environment. The purpose of this study was to evaluate the biomechanical differences in youth elite badminton players who performed FLS in activities with varying levels of predictability. Using intra-individual analysis, this study was able to show significant differences existed between trials in several of the dependent variables, which suggests that practice activities where the shuttlecock is fed from a predictable location with a lower requirement for VSB (e.g., from a stationary feeding position) may cause poor skill acquisition and development through maladaptive learning behaviours [9]. The participants in the current study were developing youth badminton players and results suggest that coaches who wish to promote effective skill acquisition may want to reevaluate their use of predictable practice routines that contain VSB different to that found in competitive play.

Participants' $\mathrm{FH}$ and $\mathrm{BH}$ dominant wrist and shoulder heights progressively lowered as the trials became less predictable. In fact, the greatest difference found in wrist height and shoulder height between handfeed and match play trials reached over 400mm (FH Wrist Height). 
Table 1 Mean trial scores, F scores, and pairwise comparisons for all trials.

\begin{tabular}{|c|c|c|c|c|c|c|c|}
\hline Dependent Variable & Handfeed Trial Mean & Multifeed Trial Mean & $\begin{array}{c}\text { Match Play Trial } \\
\text { Mean }\end{array}$ & F Score & $\begin{array}{c}\text { Handfeed \& } \\
\text { Multifeed } \\
\text { Comparison }\end{array}$ & $\begin{array}{l}\text { Handfeed } \\
\text { \& Match } \\
\text { Play } \\
\text { Compariso } \\
\text { n }\end{array}$ & $\begin{array}{l}\text { Multifeed \& Match } \\
\text { Play Comparison }\end{array}$ \\
\hline FH Wrist Height (mm) & $1201.13 \pm 116.94$ & $969.96 \pm 149.90$ & $793.87 \pm 61.58$ & $F(2,22)=45.95, p<0.001$ & $p=0.002$ & $p<0.001$ & $p=0.002$ \\
\hline BH Wrist Height (mm) & $1121.62 \pm 141.46$ & $893.86 \pm 121.30$ & $770.52 \pm 134.69$ & $F(2,22)=54.74, p<0.001$ & $\mathrm{p}<0.001$ & $\mathrm{p}<0.001$ & $p=0.02$ \\
\hline FH Shoulder Height (mm) & $1204.44 \pm 100.58$ & $1092.20 \pm 106.42$ & $955.47 \pm 71.22$ & $F(2,22)=53.95, p<0.001$ & $p=0.005$ & $p<0.001$ & $p<0.001$ \\
\hline BH Shoulder Height (mm) & $1223.51 \pm 97.51$ & $1065.10 \pm 94.21$ & $972.61 \pm 104.50$ & $F(2,22)=71.40, p<0.001$ & $\mathrm{p}<0.001$ & $\mathrm{p}<0.001$ & $p=0.014$ \\
\hline FH Trunk Angle $\left(^{\circ}\right)$ & $9.73 \pm 7.13$ & $17.72 \pm 7.05$ & $31.64 \pm 5.88$ & $F(2,22)=39.50, p<0.001$ & $p=0.031$ & $\mathrm{p}<0.001$ & $p<0.001$ \\
\hline $\mathrm{BH}$ Trunk Angle $\left(^{\circ}\right)$ & $10.08 \pm 7.69$ & $13.21 \pm 5.60$ & $17.61 \pm 8.78$ & $F(2,22)=8.42, p=0.002$ & $\mathrm{P}=0.133$ & $\mathrm{P}=0.019$ & $\mathrm{P}=0.098$ \\
\hline FH Hip Joint Angle $\left(^{\circ}\right)$ & $124.26 \pm 10.81$ & $109.15 \pm 18.53$ & $85.98 \pm 9.72$ & $F(2,22)=40.19, p<0.001$ & $P=0.04$ & $\mathrm{p}<0.001$ & $p=0.001$ \\
\hline BH Hip Joint Angle $\left(^{\circ}\right)$ & $126.49 \pm 15.45$ & $101.80 \pm 12.28$ & $90.48 \pm 15.24$ & $F(2,22)=44.25, p<0.001$ & $\mathrm{p}<0.001$ & $\mathrm{p}<0.001$ & $\mathrm{P}=0.079$ \\
\hline FH Knee Joint Angle $\left(^{\circ}\right)$ & $148.02 \pm 12.88$ & $143.84 \pm 15.34$ & $132.91 \pm 8.82$ & $F(2,22)=8.79, p=0.002$ & $\mathrm{P}=>0.999$ & $P=0.001$ & $\mathrm{P}=0.033$ \\
\hline BH Knee Joint Angle $\left(^{\circ}\right)$ & $151.20 \pm 13.85$ & $135.30 \pm 12.48$ & $133.97 \pm 12.89$ & $F(2,22)=13.71, p<0.001$ & $P=0.002$ & $P=0.002$ & $P>0.999$ \\
\hline FH Wrist Velocity $(\mathrm{mm} / \mathrm{s})$ & $2363.94 \pm 425.37$ & $2754.69 \pm 414.08$ & $3356.43 \pm 806.11$ & $F(2,22)=7.84, p=0.003$ & $\mathrm{P}=0.104$ & $P=0.013$ & $P=0.204$ \\
\hline BH Wrist Velocity (mm/s) & $2491.48 \pm 484.00$ & $2685.02 \pm 416.41$ & $3248.68 \pm 478.86$ & $F(2,22)=7.60, p=0.003$ & $P=0.579$ & $P=0.035$ & $P=0.049$ \\
\hline FH Knee Velocity (mm/s) & $2219.34 \pm 618.25$ & $2112.13 \pm 639.39$ & $1812.12 \pm 473.14$ & $F(2,22)=1.926, p=0.169$ & $p>0.999$ & $P=0.272$ & $P=0.417$ \\
\hline BH Knee Velocity (mm/s) & $2332.61 \pm 656.65$ & $1841.73 \pm 563.20$ & $1993.65 \pm 683.97$ & $F(2,22)=3.114, p=0.105$ & $\mathrm{P}=0.186$ & $P=0.316$ & $p>0.999$ \\
\hline FH Ankle Velocity (mm/s) & $911.48 \pm 568.21$ & $931.77 \pm 714.79$ & $719.25 \pm 477.78$ & $F(2,22)=1.243, p=0.308$ & $p>0.999$ & $P=0.29$ & $\mathrm{P}=0.486$ \\
\hline BH Ankle Velocity $(\mathrm{mm} / \mathrm{s})$ & $1294.49 \pm 1069.82$ & $710.92 \pm 626.16$ & $786.83 \pm 680.38$ & $F(2,22)=2.644, p=0.094$ & $\mathrm{P}=0.117$ & $P=0.448$ & $p>0.999$ \\
\hline FH Knee Joint Angular Velocity ( $\%$ s) & $-293.34 \pm 195.28$ & $-272.76 \pm 179.00$ & $266.04 \pm 94.98$ & $F(1.35,14.86)=0.123, p=0.804$ & $p>0.999$ & $p>0.999$ & $p>0.999$ \\
\hline BH Knee Joint Angular Velocity (\%/s) & $-218.90 \pm 202.58$ & $-236.88 \pm 116.36$ & $-250.74 \pm 166.41$ & $F(2,22)=0.122, p=0.886$ & $p>0.999$ & $p>0.999$ & $p>0.999$ \\
\hline
\end{tabular}


There was also a progressive decrease in knee and hip joint angle, which indicates lunge distance increased as trials became less predictable. Compared to previous lab-based kinematic analysis of lunge technique in adults [26], results from the current study returned far higher degrees of knee joint angle from all trials but similar hip joint angles for the match play trial. Forward lunge differences have been stated to not be age related [27], therefore, it is unclear in this study why youth badminton players may have larger knee joint angles than their adult counterparts. A larger lunge step with a lower shuttlecock racket strike position in the match play trial suggests a delay in lunge initiation, which confirms previous badminton research that states the importance of VSB [16]. During the more predictable handfeed and multifeed trials, where participants performed less VSB, participants were able to initiate an earlier movement and racket strike the shuttlecock at a higher position giving the potential for higher shot success [28]. Therefore, because of the quicker initiation of the lunge movement due to the requirement for less VSB, the handfeed and multifeed trials were producing significantly different kinematic FLS on shuttlecock racket strike when compared with match play trials where players would racket strike the shuttlecock from a significantly lower position while in a deeper lunge. Essentially, predictable routines caused players to practice FLS from a higher position that is unrealistic to competitive match play conditions.

Trunk angles were far less than reported in previous research [26] and may be an indicator of differences between youth and adult players. A forward trunk lean on the $\mathrm{FH}$ side significantly increased as predictability decreased, while forward trunk lean on the $\mathrm{BH}$ side only significantly increased between handfeed and match play trials. The increased forward trunk lean position confirms participants were having to reach further forward to racket strike the shuttlecock in the match play trial, potentially due to the delay in initiating the FLS. A decrease in forward trunk lean during more predictable practice routines might have a further impact upon muscle strengthening and injury prevention [29]. Increased forward trunk lean in badminton lunging has been found to engage greater use of knee extensor and ankle plantar flexor muscles [30]. Therefore, practicing with predictable routines may cause decreased muscle conditioning in the dominant quadricep and calf muscles. Also, the lack of forward trunk lean in predictable practice routines may cause deconditioning of core stabilising muscles, which are essential for supporting the trunk in forward leaning badminton activity [31].

Knee joint angular velocity provided an indicator for the temporal differences in the FLS [21]. For example, an early dominant foot landing will see greater deceleration of the knee joint at shuttlecock racket strike. Current study findings found there to be no difference in knee joint angular velocity across trials, which suggests the timing of dominant foot landing and shuttlecock racket strike were similar. The similarity in knee joint angular velocity across all trials suggest that players were still able to execute their FLS technique successfully. This was also supported by knee and ankle velocities being non-significant between trials. If participants had differences between dominant foot landing and shuttlecock racket strike, for example in the match play trial, then this might indicate the delayed movement caused the participant to change their FLS timing and technique, but this was not the case. Therefore, similarity in dominant foot landing and shuttlecock racket strike should not be an indicator for comparability between predictable practice routines and the competitive match play environment. Rather, the focus should be on the lunge depth and subsequent height of the body. Thus, when designing predictable practice routines with limited VSB, coaches should not rely on the success of shot completion as an indicator that the competitive FLS is simulated.

Importantly, VSB was controlled for in this study by the location where the shuttlecock was fed from. In the multifeed trial, participants knew where to look for the shuttlecock and the way in which the coach would play the shot (see figure 3d), but in the match play trial the participants had to search for the shuttle while being guided by opponent attributes [18]. The significant differences found between the dependent variables in the multifeed and match play trials (i.e., $\mathrm{FH}$ wrist height, $\mathrm{BH}$ wrist height, $\mathrm{FH}$ shoulder height, $\mathrm{BH}$ shoulder height, $\mathrm{FH}$ trunk angle, $\mathrm{FH}$ hip joint angle, FH knee joint angle, and $\mathrm{BH}$ wrist velocity) provide strong evidence that VSB is a key element of the FLS. An example of the technical differences in the FLS execution between multifeed and match play trials can be seen with $\mathrm{FH}$ wrist height where a mean difference of $176.09 \mathrm{~mm}$ was recorded. The FLS requires fine motor control in the racket hand to successfully execute different shots [32] so feeding routines that cause the shot to be practiced from a lower position will not be representative of the FLS experienced in competitive play. Therefore, skill acquisition and development of the FLS using 
predictable feeding practice routines without comparable competitive VSB, may cause maladaptive learning behaviours. Results from the current study recommend that coaches include competitive VSB during all practice routines.

\subsection{Limitations and Future Research}

Despite this study having equal [17] or higher participant numbers $[4,14,15]$ compared to previous research in this field, adequate level of power for a medium effect size was not achieved using G*Power. However, the specific population evaluated in this study restricted high participant numbers. Future research using a similar experimental design would require approximately 28 participants. This study reported VSB to be highly influential in badminton FLS. A direct evaluation of VSB using eye tracking technology [33] during practice and match situations would provide further comparison. The current study assessed the FLS and future research could be applied to other badminton movements. Researchers with access to shuttlecock feeding machines should continue to assess their impact on skill acquisition and development compared to unpredictable practice.

\section{Conclusion}

In the badminton practice environment, it is commonplace to see predictable feeding routines where the feeder holds a stationary position. Also, recent advances in technology have seen the use of shuttlecock feeding machines in badminton. The results from this study suggest that predictable feeding drills (e.g., the feeder does not move position) will significantly alter the biomechanics of the FLS. Therefore, skill acquisition and development of the FLS will be compromised. This study highlights the need for coaches to move away from predictable feeding practice routines due to lower levels of VSB. It is recommended that coaches use practice routines where the feeding player is mobile and simulates the movements seen in competitive play where full VSB is required.

\section{References}

[1] M. Phomsoupha, G. Laffaye, The Science of Badminton: Game Characteristics, Anthropometry, Physiology, Visual Fitness and Biomechanics, Sports Medicine, 45(4) (2015) 473-495. [DOI] [PubMed]
[2] V. Correia, J. Carvalho, D. Araújo, E. Pereira, K. Davids, Principles of Nonlinear Pedagogy in Sport Practice, Physical Education and Sport Pedagogy, 24(2) (2019) 117-132. [DOI]

[3] K. Davids, D. Araújo, L. Vilar, I. Renshaw, R. Pinder, An Ecological Dynamics Approach to Skill Acquisition: Implications for Development of Talent in Sport, Talent Development \& Excellence, 5(1) (2013) 21-34.

[4] L. Krause, D. Farrow, T. Buszard, R. Pinder, M. Reid, Application of Representative Learning Design for Assessment of Common Practice Tasks in Tennis, Psychology of Sport and Exercise, 41 (2019) 36-45. [DOI]

[5] R. J. Martindale, D. Collins, A. Abraham, Effective Talent Development: The Elite Coach Perspective in UK Sport, Journal of Applied Sport Psychology, 19(2) (2007) 187-206. [DOI]

[6] Level 2 - BWF Development, Development.bwfbadminton.com. (2021), https://development.bwfbadminton.com/coach es/level-2

[7] M. U. Aslam, A. Bashir, W. U. Draz, H. M. M. Raja, Optimized Shuttlecock Propulsion Machine to Facilitate Badminton Training, 2019 International Conference on Electrical, Communication, and Computer Engineering, (2019) 1-6. [DOI]

[8] A. P. G. De Alwis, C. Dehikumbura, M. Konthawardana, T. D. Lalitharatne, V. P. C. Dassanayake, Design and Development of a Badminton Shuttlecock Feeding Machine to Reproduce Actual Badminton Shots, 2020 5th International Conference on Control and Robotics Engineering, (2020) 73-77. [DOI]

[9] R. A. Pinder, K. Davids, I. Renshaw, D. Araújo, (2011) Manipulating Informational Constraints Shapes Movement Reorganization in Interceptive Actions, Attention, Perception, \& Psychophysics, 73(4) 1242-1254. [DOI] [PubMed]

[10] L. Lobo, M. Heras-Escribano, D. Travieso, The History and Philosophy of Ecological Psychology, Frontiers in Psychology, 9 (2018) 2228. [DOI] [PubMed]

[11] C. T. Woods, I. McKeown, M. Rothwell, D. Araújo, S. Robertson, K. Davids, Sport Practitioners as Sport Ecology Designers: How Ecological dynamics has Progressively Changed Perceptions of Skill "Acquisition" in the 
Sporting Habitat, Frontiers in psychology, 11 (2020) 654. [DOI] [PubMed]

[12] P. R. Browne, C. T. Woods, A. J. Sweeting, S. Robertson, (2020) Applications of a Working Framework for the Measurement of Representative Learning Design in Australian Football, PloS one, 15(11) e0242336. [DOI] [PubMed]

[13] I. Renshaw, J. Y. Chow, K. Davids, J. Hammond, (2010). A Constraints-Led Perspective to Understanding Skill Acquisition and Game Play: A Basis for Integration of Motor Learning Theory and Physical Education Praxis?, Physical Education and Sport Pedagogy, 15(2) 117-137. [DOI]

[14] D. Orth, K. Davids, D. Araújo, I. Renshaw, P. Passos, Effects of a Defender on Run-Up Velocity and Ball Speed When Crossing a Football, European Journal of Sport Science, 14(sup1) (2014) S316-S323. [DOI] [PubMed]

[15] S. Barris, K. Davids, D. Farrow, Representative Learning Design in Springboard Diving: Is DryLand Training Representative of a Pool Dive?, European Journal of Sport Science, 13(6) (2013) 638-645. [DOI] [PubMed]

[16] D. Alder, P. R. Ford, J. Causer, A. M. Williams, The Coupling Between Gaze Behavior and Opponent Kinematics During Anticipation of Badminton Shots, Human Movement Science, 37 (2014) 167-179. [DOI] [PubMed]

[17] A. D. Gorman, M. A. Maloney, Representative design: Does the Addition of a Defender Change the Execution of a Basketball Shot?, Psychology of Sport and Exercise, 27 (2016) 112-119. [DOI]

[18] J. M. Wolfe, T. S. Horowitz, Five Factors That Guide Attention in Visual Search, Nature Human Behaviour, 1(3) (2017) 1-8. [DOI]

[19] J. K. Witt, M. A. Riley, Discovering Your Inner Gibson: Reconciling Action-Specific and Ecological Approaches to Perception-Action, Psychonomic Bulletin \& Review, 21(6) (2014) 1353-1370. [DOI] [PubMed]

[20] L. Fu, F. Ren, J. S. Baker, Comparison of Joint Loading in Badminton Lunging Between Professional and Amateur Badminton Players, Applied Bionics and Biomechanics, 2017, (2017) 1-8. [DOI] [PubMed]

[21] G. Kuntze, N. Mansfield, W. Sellers, A Biomechanical Analysis of Common Lunge
Tasks in Badminton, Journal of Sports Sciences, 28(2) (2010) 183-191. [DOI] [PubMed]

[22] R. Valldecabres, C. A. Casal, J. G. C. Chiminazzo, A. M. De Benito, Players' on-Court Movements and Contextual Variables in Badminton World Championship, Frontiers in Psychology, 11 (2020) 1567. [DOI] [PubMed]

[23] L. Yu, Q. Mei, N. I. Mohamad, Y. Gu, J. Fernandez, An Exploratory Investigation of Patellofemoral Joint Loadings During Directional Lunges in Badminton, Computers in Biology and Medicine, 132 (2021) 104302. [DOI] [PubMed]

[24] Y. Le Meur, C. Hausswirth, F. Natta, A. Couturier, F. Bignet, P. P. Vidal, A Multidisciplinary Approach to Overreaching Detection in Endurance Trained Athletes, Journal of Applied Physiology, 114(3) (2013) 411-420. [DOI] [PubMed]

[25] S. J. Maloney, Review of the Badminton Lunge and Specific Training Considerations, Strength \& Conditioning Journal, 40(4) (2018) 7-17. [DOI]

[26] A. M. Nadzalan, S. H. Azmi, N. I. Mohamad, J. L. F. Lee, K. Tan, C. Chinnasee, Kinematics Analysis of Dominant and Non-Dominant Lower Limb During Step and Jump Forward Lunge in Badminton, Journal of Fundamental and Applied Sciences, 10(3S) (2018) 232-242.

[27] S. Jönhagen, P. Ackermann, T. Saartok, Forward Lunge: A Training Study of Eccentric Exercises of the Lower Limbs, The Journal of Strength \& Conditioning Research, 23(3) (2009) 972-978. [DOI] [PubMed]

[28] W. Gawin, C. Beyer, H. Hasse, D. Büsch, How to Attack the Service: An Empirical Contribution to Rally Opening in World-Class Badminton Doubles, International Journal of Performance Analysis in Sport, 13(3) (2013) 860-871. [DOI] [PubMed]

[29] M. T. Huang, H. H. Lee, C. F. Lin, Y. J. Tsai, J. C.Liao, How Does Knee Pain Affect Trunk and Knee Motion During Badminton Forehand Lunges?, Journal of Sports Sciences, 32 (2014) 690-700. [DOI] [PubMed]

[30] C. F. Lin, S. H. Hua, M. T. Huang, H. H. Lee, J. C. Liao, Biomechanical Analysis of Knee and Trunk in Badminton Players with and Without Knee Pain During Backhand Diagonal Lunges, 
Journal of Sports Sciences, 33(14) (2015) 1429-1439. [DOI] [PubMed]

[31] M. Phomsoupha, G. Laffaye, Injuries in Badminton: A Review Blessures en badminton: une revue, Science \& Sports, 35(4) (2020) 189-199. [DOI]

[32] Y. C. Hsueh, Y. Y. Chen, K. M. Pan, C. L. Tsai, Biomechanical Analysis of Badminton Forehand Net Shots, In 30th International Conference of Biomechanics in Sports, (2012) 256-259.

[33] R. M. Discombe, S. T. Cotterill, Eye Tracking in Sport: A guide for New and Aspiring Researchers, Sport \& Exercise Psychology Review, 11(2) (2015) 49-58.

\section{Acknowledgement}

The authors gratefully acknowledge the financial support from the Hampshire Badminton Association.

\section{Funding}

No funding was received for conducting this study.

\section{Authors Contribution}

Dr S.M. Smith led this research project and wrote this manuscript. E. Tasker provided principle support on data collection and analysis. E. Paine led data collection with support from T.M. Hughes and C. Heiden. O. Baczala provided badminton specific data collection support.

\section{Conflict of interest}

The authors have no conflicts of interest to declare that they are relevant to the content of this article.

\section{Informed Consent}

Written consent was obtained from both the participants and their parents

\section{Ethics Approval}

This study was approved by Institutional Review Board at University of Winchester.

\section{Availability of data and material}

No additional data are available.

\section{Does this article screened for similarity?}

Yes

\section{About The License}

(C) The author(s) 2022. The text of this article is open access and licensed under a Creative Commons Attribution 4.0 International License 